\title{
Development of technology for a fermented drink with cancer protective properties
}

\author{
Tatyana Belyakova ${ }^{1,{ }^{*}}$, Lyudmila Zabodalova ${ }^{1}$, and Ekaterina Antonceva ${ }^{2}$ \\ ${ }^{1}$ Saint Petersburg National Research University of Information Technology, Mechanics and Optics \\ (ITMO University), 191002, Lomonosova str. 9, Saint Petersburg, Russia \\ ${ }^{2}$ Saint-Petersburg State Institute of Technology, 190013, Moskovsky pr., 26, Saint-Petersburg, Russia
}

\begin{abstract}
Some plant sources of biologically active substances particularly cruciferous vegetables, both in native form and as extracts, display effects towards carcinogenesis that make these food sources cancer protective and recurrence preventive agents. In the currently developing tendency towards functional foodstuffs like dairy products targeted at nutritional-dependent diseases, development of a dairy beverage containing sulforaphane deserves attention. Creating a recipe for such a product includes: selecting a plant sulforaphane source, testing its compatibility with the milk base, choosing the addition dosage and methods and experimental beverage preparation. In pursuit of these goals in the study, firstly, turnip (Brassica rapa l.) root, an affordable sulforaphane provider, was chosen. Glucoraphanin content in various turnip varieties was determined and the Golden Ball variety selected. Sulforaphane was introduced to milk in turnip purée and aqueous extract forms. The effect of purée/extract dosage on milk fermentation process and the final product's quality parameters was investigated. Both purée and extract containing products had a pleasant taste with a vegetable note and uniform consistency. Sulforaphane content in $200 \mathrm{~g}$ beverage servings was $(23.5 \pm 2.1) \mathrm{mg}$ and $(29.5 \pm 0.5)$ $\mathrm{mg}$ (extract), both values approximately one tenth of therapeutic dose estimates, which is enough for cancer preventive purposes.
\end{abstract}

\section{Introduction}

Prophylaxis and treatment of nutrition related cancer has been a leading research topic in many institutions for decades. The World Cancer Research Fund has developed recommendations for nutrition-related cancer treatment [1]. Besides other sound dietary practices such as body mass control, the use of cancer protective food grade substances in diet can lower the risk of malignity development. These natural agents are biologically active (bioactive) substances of plant origin [2].

In 2013, the World Health Organization published a list of foods capable of stopping cancer cell growth. The list includes cruciferous plants (broccoli, cauliflower, kohlrabi, turnip), soy, onion, garlic and much more [3]. Several research teams found relationships between an increase in cruciferous vegetable consumption and a decrease in the onset of

\footnotetext{
* Corresponding author: tnbelyakova517@gmail.com
} 
breast, colon, lung, pancreas and prostate cancer [4-9]. Moreover, at least one serving of cruciferous vegetables per week reduces the risk of breast, oral cavity and pharynx, esophagus, intestine, and kidney cancer compared to the same basal diet without cruciferous vegetables [10].

It is recognized that an important role in fighting malignities belongs to supportive care that relies on various methods to sustain the patient throughout the period of cancer treatment [1, 11] lowering the relapsing incidence and complication risks [12]. Recent studies connect consumption of some bioactive substances with mutagen destruction, leading to lowered recurrence and reduction of both metastasis and therapy resistance. It is believed that the substances in question inhibit enzymes that are pro-carcinogen activators, activate enzymes that eventually destroy the mutagen and mediate a number of antitumor pathways such as apoptosis activation [13]. Sulforaphane is one such protective substance.

Sulphoraphane, being an antibacterial substance, is an infection protector in plants, where the compound is mainly encountered in the form of a glucosinolate, glucoraphanin. As bacterial infection is often caused by physical damage of a plant's tissues, cell wall breaching initiates glucoraphanin conversion to sulforaphane by the enzyme myrosinase [2].

One of the main dietary sources of sulforaphane is the group of cruciferous vegetables that are much more often cooked andor mechanically processed than eaten raw, so the conditions for glucoraphanin conversion to sulforaphane are met. Besides cabbage, broccoli, mustard etc., the family Cruciferae, or more precisely Brassicaceae, includes turnip (Brassica rapa), an annual or biennial herbaceous plant valued for its root, a rich source of various diet components: vitamins, dietary fibre and glucosinolates, including the precursor for sulforaphane [2]. Turnip is generally affordable and can be freely cultivated.

The presence of sulforaphane in everyday diet could be increased by including processed turnip into foods such as dairy products. However, using a turnip filler in drink preparation may cause complications such as off-flavours, alteration of structural properties and fermentation issues. Therefore, investigating the possible effects of therapeutic sulforaphane dose addition (by introducing processed turnip root) to a fermented cow's milk beverage deserves attention.

\section{Materials and methods}

The ingredients used in the fermented drink recipes screened in this research were:

- raw cow's milk complying to the Russian government standard 31449-2013;

- fresh young turnip of Dunyasha, Golden Ball, Petrovskaya-1 and Zolotoy Shar varieties (Russian government standard 32791-2014);

- aqueous sulforaphane extract;

- milk and vegetable filler mix;

- clots formed during fermentation of the mix.

Developing the composition of fermented drink recipes took two directions: a) adding turnip purée to milk; b) extracting sulforaphane and introducing the extract to milk.

Heat treatment during the preparation of turnip puree had to be sparing to avoid glucoraphanin destabilization, protein denaturation, vitamin destruction and melanoidin formation. In this regard, three options were tested:

- boiling raw turnip roots until a noticeable increase in palpability;

- baking roots in a Snol convective oven at $(150 \pm 5)^{\circ} \mathrm{C}$ for $60-80 \mathrm{~min}$;

- steaming in the same oven at $(95 \pm 5)^{\circ} \mathrm{C}$ for $30 \mathrm{~min}$ in a lidded glass dish with a small amount of water. 
Glucoraphanin content in each sample after the appropriate heat treatment was determined with Osin's method [14]. The determination method included: air drying of puréed roots while retaining fat; dissolution of the dry purée with concentrated alkali for sulfidization; addition of concentrated hydrochloric acid for hydrogen sulfide formation; distillation of the mixture in a flask with absorber. Absorbed hydrogen sulfide was quantified with the iodometric method.

Sulforaphane extract was prepared in the following manner. Fresh turnip roots were freezed to $(-20 \pm 1)^{\circ} \mathrm{C}$ for $12 \mathrm{~h}$, thawed at room temperature for $6 \mathrm{~h}$, then again freezed for $12 \mathrm{~h}$ and thawed for $6 \mathrm{~h}$ to increase extract yield from plant cells. Then the roots were puréed with a kitchen blender, kept in a LOIP-2 thermostatic water bath at $(60 \pm 1){ }^{\circ} \mathrm{C}$ for $60 \mathrm{~min}$ to promote glucoraphanin conversion to sulforaphane and dried to a constant mass at $(80 \pm 2)^{\circ} \mathrm{C}$. Wet and dried purée samples were weighed with Shimadzu precision scales.

Weighed samples of dried purée were put in sterile $250 \mathrm{~mL}$ Erlenmeyer flasks filled with distilled water and kept in a Medtekhnika thermostat for $24 \mathrm{~h}$ at $(30 \pm 1)^{\circ} \mathrm{C}$ with constant magnetic stirring. The resulting aqueous extract was vacuum filtered through a Whatman no. 2 paper filter in a ceramic Büchner funnel and collected in a $500 \mathrm{~mL}$ Bunsen flask. During filtration the residual pressure under the funnel was kept at 0.1 bar and the temperature at room level. Glucoraphanin content in the extract was measured by the method described above.

The next experimental stage was designed to compare samples made by either fermenting milk with the purée/extract added or adding the purée/extract after the fermentation.

When determining the rational dose of sulforaphane source to be added, extract dosage was based on the previously published research, while purée dosage was calculated from the active sulforaphane dose extrapolated by Herr et al. (2010) since no clinical trial have been conducted so far. The boundary concentration to inhibit human cancer xenografts in mice was $4.4 \mathrm{mg} \mathrm{kg}^{-1}$ day $^{-1}$ which corresponds to a daily $308 \mathrm{mg}$ dose for a $70 \mathrm{~kg}$ human $[15,16]$.

These calculations resulted in 15, 20 and $25 \%$ dosage for turnip purée and 3.5-17.5\% dosage (in $3.5 \%$ increments) for the sulforaphane extract.

Samples of cow's milk with $2.5 \%$ fat were fermented with concentrated directlyophilized starter cultures Marino CBL 9 (Lactobacillus acidophilus bacteria) and Yo-Mix 305 LYO 250 DCU (L. delbrückii subsp. bulgaricus and Streptococcus thermophilus) for $78 \mathrm{~h}$ at $(40 \pm 2)^{\circ} \mathrm{C}$ until 85.5 degree titratable acidity was achieved.

Titratable acidity was determined according to the Russian government standard 3624-92, and ph by the standard 32892-2014.

The moisture holding capacity of the clot in the fermented product was determined by centrifuging $10 \mathrm{~cm} 3$ destroyed clot in a $15 \mathrm{~cm} 3$ centrifuge tube at $1000 \mathrm{~min}^{-1}$ for $5 \mathrm{~min}$, decanting the released serum into a graduated $15 \mathrm{~cm} 3$ centrifuge tube, centrifuging the sample for another $5 \mathrm{~min}$ and decanting until the total centrifugation time was $30 \mathrm{~min}$. The amount of serum released was used to measure the effect of extract dose on moisture retention.

Seeing as most dairy products are thixotropic materials, the samples' structure resilience to mechanical stress needed assay, so that the thixotropic properties could be determined. The appropriate rheological studies were done with Rheotest 2 device. Dynamic viscosity of the samples was measured at $15 \mathrm{~s}$ intervals during a 2 min exposure to a homogenous shear force field with a constant $145.8 \mathrm{~s}^{-1}$ shear velocity gradient.

The parameters were calculated by the formula (1-3):

$$
\Pi_{\eta}=\frac{\eta_{I}-\eta_{D}}{\eta_{I}} \times 100 \%
$$




$$
\begin{gathered}
\mathrm{B}_{\eta}=\frac{\eta_{R}}{\eta_{I}} \times 100 \% \\
\frac{\eta_{I}}{\eta_{D}}
\end{gathered}
$$

$\Pi_{\eta}$ - viscosity loss coefficient $\Pi_{\eta}$ (capital letter $p i$ with subscript eta);

$\eta_{I}$ - dynamic viscosity of the intact clot;

$\eta_{D}$ - dynamic viscosity after 2 min exposure to shear destruction;

$\mathrm{B}_{\eta}$ - structure reforming coefficient on relaxation $\mathrm{B}_{\eta}$ (capital beta with subscript eta);

$\eta_{R}$ - dynamic viscosity of recovered structure (15 min after destruction);

$\eta_{I} / \eta_{D}$ - coefficient of mechanical stability.

Sample organoleptics were evaluated according to the RussianISO standard 22935-32011 and expressed in scores from 1 to 5 on the following scale.

All experiments and measurements (besides the hedonistic appraisal described below) were done in triplicate. Experimental errors were calculated at $95 \%$ confidence level.

Table 1. Scale of organoleptic properties differentiation.

\begin{tabular}{|c|c|}
\hline Score & Verbal description \\
\hline 5 & No deviation from the previously established requirements \\
\hline 4 & Minimum deviation from the previously established requirements \\
\hline 3 & Noticeable deviation from the previously established requirements \\
\hline 2 & Significant deviation from the previously established requirements \\
\hline 1 & Grossly significant deviation from the previously established requirements \\
\hline
\end{tabular}

Sensory evaluation was performed by 6 trained panellists using the scale above to judge the degree of compliance to the following quality requirements (Table 1):

- appearance and consistency: must be homogeneous, with the clot intact, slightly viscous, possibly with undissolved particles belonging to the added material;

- taste and odour: clean, sour-milk, no off-flavours besides the ones belonging to the added material;

- transparency and colour: milky; either white or characteristic of the added material.

\section{Results and discussion}

In the first experimental stage it was found that the variety most rich in glucoraphanin $(55.0 \pm 3.1 \mathrm{mg}$ per $100 \mathrm{~g})$ is the Golden Ball turnip, which was used from here on after.

The effect of heat treatment on glucoraphanin content (Table 2) was the lowest in steamed roots, so this treatment mode was used in subsequent experimental stages.

Table 2. Glucoraphanin content in turnip roots subjected to different modes of heat treatment.

\begin{tabular}{|c|c|}
\hline Heat treatment applied & Glucoraphanin $(\mathrm{mg}$ per $100 \mathrm{~g})$ \\
\hline Boiled & $19.0 \pm 2.5$ \\
\hline Oven baked & $25.0 \pm 2.5$ \\
\hline Steamed & $30.0 \pm 2.5$ \\
\hline
\end{tabular}

The following experimental stage had possible ways of sulforaphane source addition compared. When turnip purée was added to fermented milk, the samples had unstable consistency and non-uniform taste. In contrast, the samples with the purée added before fermentation had a notably even filler distribution that led to uniform structure and taste. 
Fig. 1 shows the dynamics of titrated acid accumulation in the samples. The initial acidity in the control and experimental samples was approximately equal at 20-21 degrees. After $1.5 \mathrm{~h}$ of fermentation, a more intense increase in acidity was observed in the samples with the puree filler. The ripening process was clearly more intensive in the samples with a $25 \%$ mass fraction of purée than in control, probably due to the additional fermentable sugars introduced with the filler and consumed by starter microflora (other differences insignificant at $95 \%$ confidence level). The change in $\mathrm{pH}$ displays a similar trend.

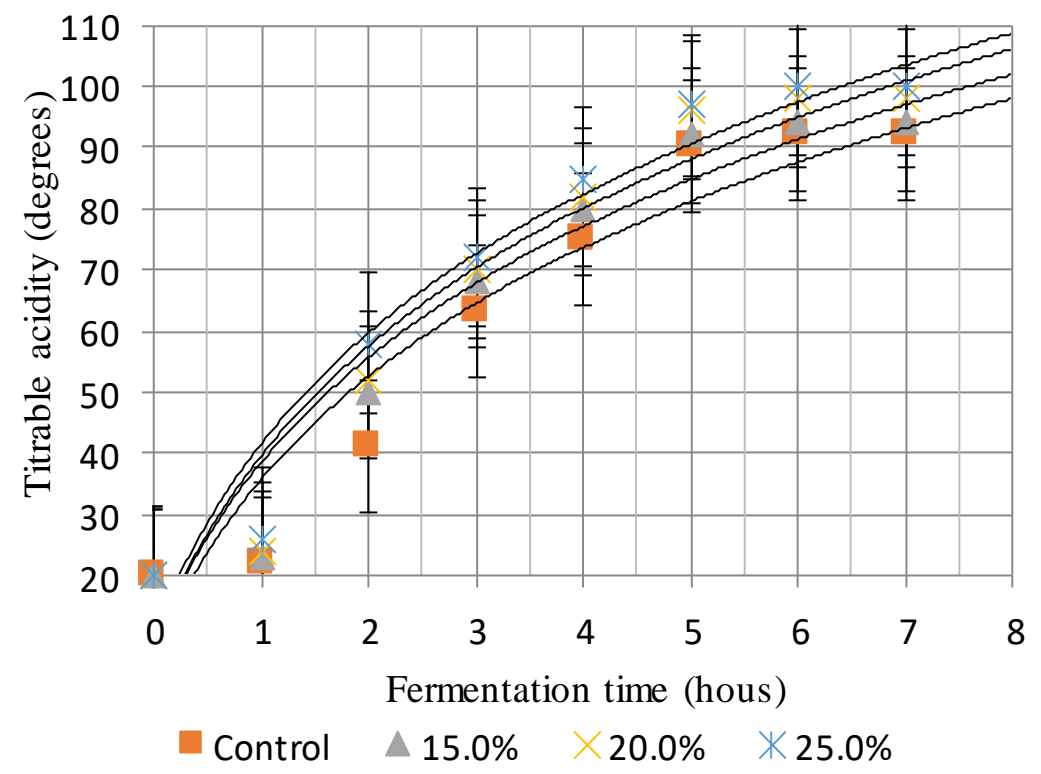

Fig. 1. The samples' titratable acidity change over fermentation time.

The effect of purée addition on the product's consistency was measured next. The intensity of serum release in samples with added purée decreased after $15 \mathrm{~min}$ of centrifugation and after $25 \mathrm{~min}$ in the control samples. The total serum volume released at the end of experimental samples centrifugation was less than the corresponding amount for the control; moreover, serum volume decreased as the filler percentage increased (Fig. 2). Filler addition thus proved to increase clot viscosity and water holding capacity as compared to the control, evidently due to the ability of turnip purée to bind free moisture. 


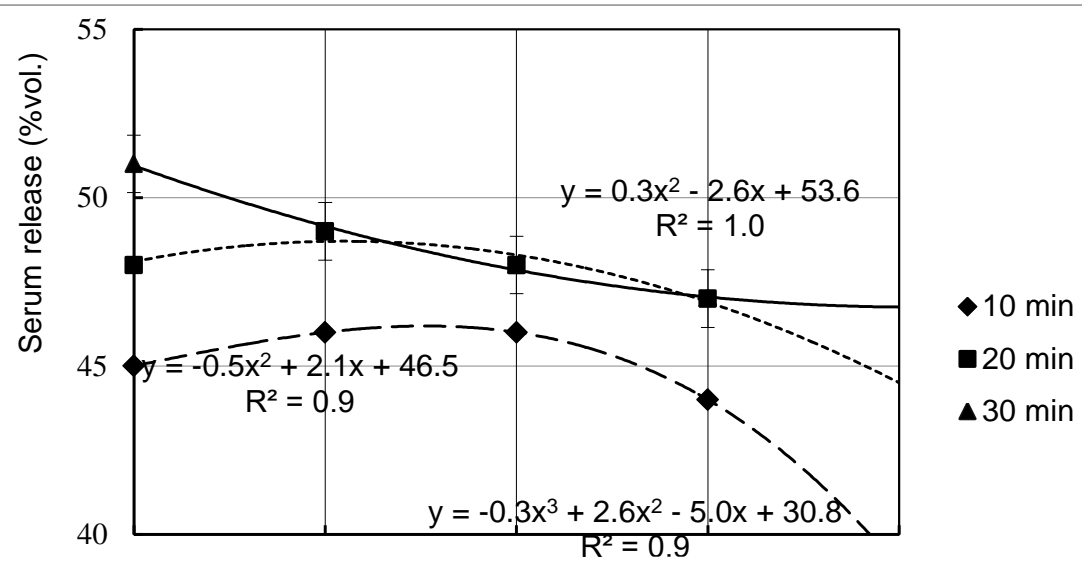

Filler added (\% mass)

Fig. 2. The effect of filler percentage on the samples' water holding capacity.

Table 3 shows the results of rheological studies characterizing the product structure's resilience to mechanical stress and the ability for structure reformation (differences between control and the other samples statistically significant at $95 \%$ confidence level, difference between samples insignificant).

Table 3. The effect of filler amount on the product's structural and mechanical properties.

\begin{tabular}{|c|c|c|c|}
\hline \multirow{2}{*}{$\begin{array}{c}\text { Filler dosage } \\
(\% \text { mass })\end{array}$} & \multicolumn{3}{|c|}{ Property } \\
\cline { 2 - 4 } & $\Pi_{\eta}(\%)$ & $\eta_{I} / \eta_{D}$ & $\mathrm{~B}_{\eta}(\%)$ \\
\hline 15.0 & $64 \pm 2$ & $2.2 \pm 0.1$ & $41 \pm 2$ \\
\hline 20.0 & $55 \pm 1$ & $2.3 \pm 0.1$ & $46 . \pm 1$ \\
\hline 25.0 & $30 \pm 1$ & $2.2 \pm 0.1$ & $53 \pm 1$ \\
\hline
\end{tabular}

Samples with $25 \%$ purée added showed lower values of the viscosity loss coefficient and higher values of the structure reforming coefficient compared to other samples. The ratio of initial viscosity to destroyed clot viscosity changed little.

In judging the samples' organoleptic properties it was found that the experimental samples differed from the control by the clot colour (pronounced yellow-orange tint attributed to the filler). All samples had a uniformly viscous consistency. Again, the samples with $25 \%$ performed the best.

Taste of the samples generally benefitted from filler addition (Fig. 3). While the samples with $15 \%$ and $20 \%$ puree added did not have a developed specific taste, the samples with $25 \%$ filler had a pleasant, moderately pronounced sweetish-spicy taste. In contrast, all panellists noted a pungent odour in every sample that contained turnip purée. 

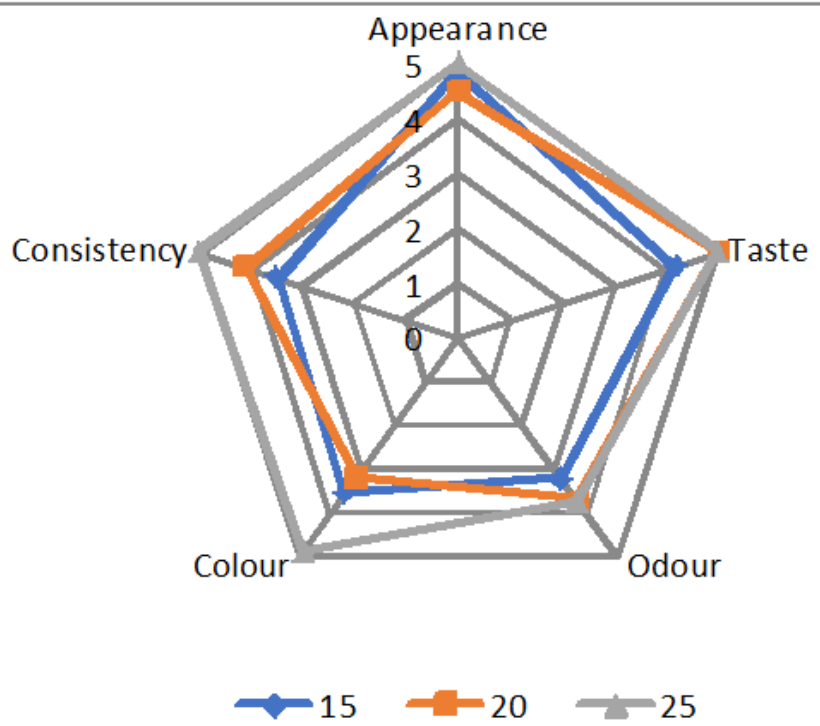

Fig. 3. Web diagram of the filler amount effect on the samples' organoleptics.

According to the best overall performance of the $25 \%$ variant, it was chosen as the basis for further recipe improvement. With this filler percentage, a $200 \mathrm{~g}$ serving of the product contained $(24.5 \pm 2.0) \mathrm{mg}$ of the active ingredient.

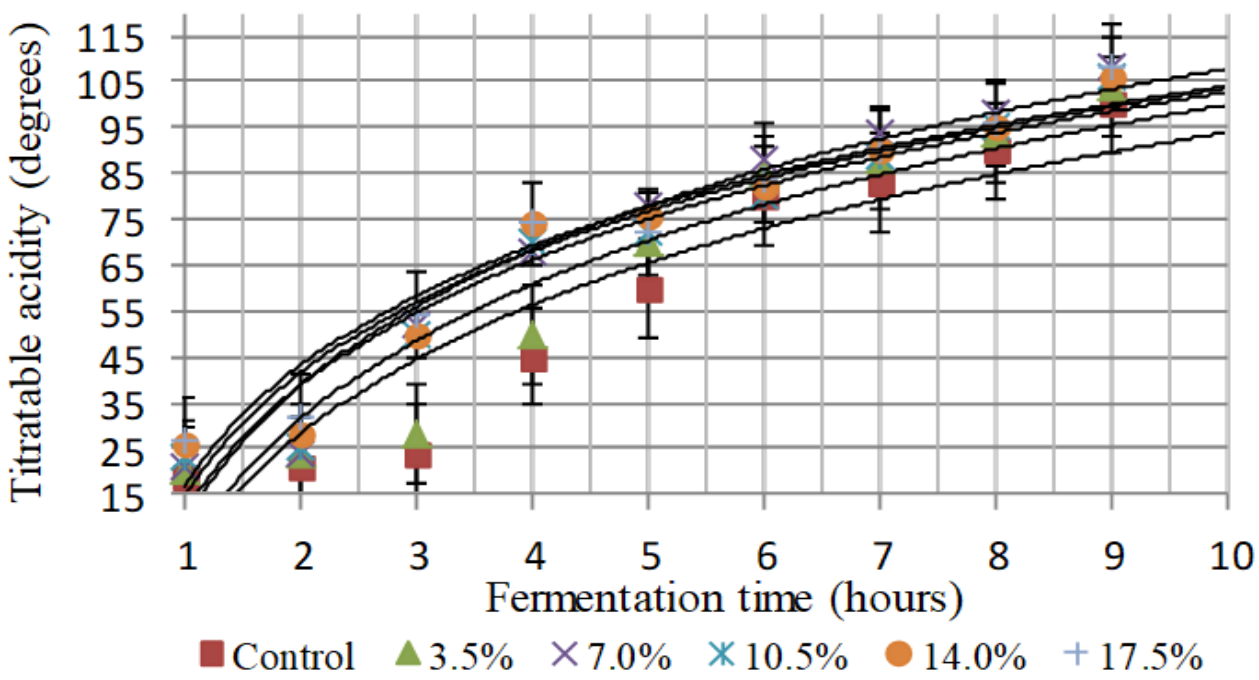

Fig. 4. Evolution of the samples' titratable acidity during fermentation.

Aqueous sulforaphane extract was further prepared with the method described in the previous section and tried in the fermented beverage preparation. The necessary content of sulforaphane was calculated according to the material balance equation taking into account its molecular weight. As a result, it was found that the content of sulforaphane in $1 \mathrm{~g}$ of dry turnip roots was $(35.2 \pm 0.4) \mathrm{mg}$, and $(0.83 \pm 0.03) \mathrm{mg}$ in $1 \mathrm{~cm} 3$ extract. The latter was added to the fermented milk at the mass percentage of $3.5-17.5 \%$ in $3.5 \%$ increments. The same characteristics as measured above for the purée-filled samples were determined in the extract-infused samples (Fig. 4 and 5, Table 5). 
Due to the milk colloidal system changes during fermentation the clot structure is formed that consumer qualities largely depend on. It is thus prudent to study the changes the clot structure would undergo in possible treatment. Table 5 demonstrates these effects (all differences besides the $\eta$ i $\eta$ d ratio statistically significant at $95 \%$ confidence level).

Table 4. The effect of extract amount on the product's structural and mechanical properties.

\begin{tabular}{|c|c|c|c|c|c|c|}
\hline \multirow{3}{*}{$\begin{array}{c}\text { Extract } \\
\text { amount } \\
(\%)\end{array}$} & \multicolumn{6}{|c|}{ Property } \\
\hline & \multirow{2}{*}{$\Pi_{\eta}(\%)$} & \multirow{2}{*}{$\eta_{I} / \eta_{D}$} & \multirow{2}{*}{$\mathbf{B}_{\boldsymbol{\eta}}(\%)$} & \multicolumn{3}{|c|}{ structure viscosity $\left(\mathrm{Pa} \cdot \mathrm{s} 10^{2}\right)$} \\
\hline & & & & intact & destroyed & restored \\
\hline control & $19 \pm 2$ & $1.2 \pm 0.1$ & $41 \pm 2$ & $57.5 \pm 0.4$ & $46.4 \pm 0.5$ & $49.4 \pm 0.5$ \\
\hline 3.5 & $11 \pm 1$ & $1.1 \pm 0.1$ & $92 \pm 1$ & $41.6 \pm 0.5$ & $37.1 \pm 0.4$ & $38.1 \pm 0.3$ \\
\hline 7.0 & $20 \pm 1$ & $1.3 \pm 0.2$ & $82 \pm 1$ & $51.5 \pm 0.4$ & $41.3 \pm 0.5$ & $42.2 \pm 0.4$ \\
\hline 10.5 & $24 \pm 2$ & $1.3 \pm 0.2$ & $79 \pm 1$ & $44.5 \pm 0.4$ & $33.9 \pm 0.4$ & $34.9 \pm 0.4$ \\
\hline 14.0 & $12 \pm 1$ & $1.1 \pm 0.1$ & $91 \pm 3$ & $38.8 \pm 0.5$ & $34.2 \pm 0.4$ & $35.3 \pm 0.4$ \\
\hline 17.5 & $9 \pm 1$ & $1.1 \pm 0.1$ & $91 \pm 2$ & $30.3 \pm 0.4$ & $27.5 \pm 0.4$ & $27.4 \pm 0.5$ \\
\hline
\end{tabular}

The rheological studies showed that extract addition decreased product consistency, making it more liquid than the control, as evidenced by a decrease in the samples' effective viscosity (with the clot intact) together with an increase in extract dosage. While neither a direct nor an adverse effect of extract dose on the properties in Table 5 could be seen, the degree of structural restoration in the samples with the highest extract dose was quite high.

All samples but the control and the samples with the least extract showed a tendency for serum reabsorption if left for $12 \mathrm{~h}$ in a regular refrigerator and then stirred. While the released serum volume slightly increased with an increase in the extract dosage, subsequent release of the 'stirred-in' serum was not observed for several hours. Based on this observation it was assumed that the clot structure was completely restored and the serum was again retained by the structure proteins and carbohydrates that make up the filler.

Determination of the clot water holding capacity by centrifugation (Table 5) showed that during the lengthy centrifugation process, the clots' ability to retain serum uniformly lowered across the experimental sample matrix (differences between control and the other samples statistically significant at $95 \%$ confidence level, difference between samples insignificant).

Table 5. The effect of extract amount on the product's structural and mechanical properties.

\begin{tabular}{|c|c|c|c|}
\hline \multirow{2}{*}{ Extract amount (\%) } & \multicolumn{3}{|c|}{ Centrifugation time } \\
\cline { 2 - 4 } & $10 \mathrm{~min}$ & $20 \mathrm{~min}$ & $30 \mathrm{~min}$ \\
\hline control & 31 & 33 & 34 \\
\hline 3.5 & 28 & 35 & 37 \\
\hline 7.0 & 29 & 39 & 39 \\
\hline 10.5 & 30 & 41 & 41 \\
\hline 14.0 & 32 & 46 & 46 \\
\hline 17.5 & 32 & 46 & 49 \\
\hline
\end{tabular}

As the change was evidently caused by the mechanical action, it could be deduced that the fermented beverage must not be subjected to vigorous mechanical processing, or otherwise should be made more resistant by an additional stabilizer.

Finally, the organoleptic characteristics of the extract-infused samples showed a positive difference from the control (Fig. 5). 


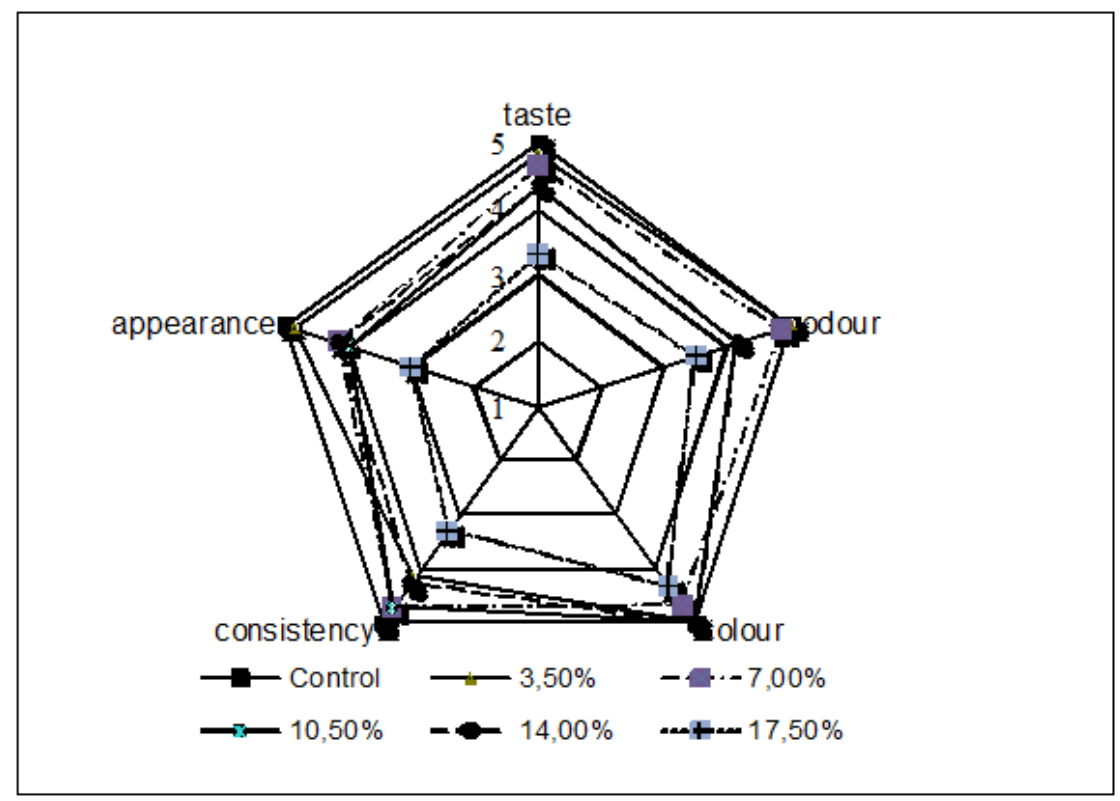

Fig. 5. Web diagram of the extract amount effect on the samples' organoleptics.

All experimental samples had a uniformly viscous consistency; however, the samples with the most extract added had a slight aftertaste of cruciferous vegetables.

\section{Conclusion}

The current study demonstrates the expediency of using turnip roots as an affordable source of sulforaphane in a fermented beverage with cancer protective properties. Based on the availability and high glucoraphanin (sulforaphane precursor) the Golden Ball variety can be recommended.

The rational mode of turnip root heat treatment to obtain purée with most active substance spared and the stage of purée introduction into the milk base were determined. Adding steamed turnip purée before fermentation proved to be the most adequate.

Turnip purée addition at $25 \%$ milk base mass gives a product without separation of serum, with optimal structural, mechanical and organoleptic properties, except for a specific odour due to the filler source. A $200 \mathrm{~g}$ serving of the beverage contained $(24.5 \pm 2.0) \mathrm{mg}$ of the active substance, making it a functional product recommended for prophylactic nutrition in all age groups.

In contrast, sulforaphane extract addition to the milk base results in a product with a uniform but less viscous consistency and more pronounced thixotropic properties, as compared to the control. The product with the highest doses of the extract had a slight aftertaste characteristic of the introduced filler, which did not significantly affect the perception of the product.

On the whole, sulforaphane extract dosage of $17.5 \%$ can be recommended for addition. Consuming a $200 \mathrm{~g}$ serving of the product with this amount of the extract amounts to $(29.1 \pm 1.0) \mathrm{mg}$ sulforaphane intake, which is about $10 \%$ of the therapeutic dose, sufficient for preventive purposes or as a nutritional support to prevent cancer relapse during supportive care. In order to increase the content of sulforaphane in the product to a therapeutic dose, extract concentration or drying can be attempted in the future. 


\section{References}

1. J. Herrstedt, International Symposium on Supportive Care in Cancer (Miami, USA, 2014)

2. T. Belyakova, O. Morozova, E. Antonceva, L. Zabodalova, E3S Web of Conferences 164, 06028 (2020) DOI: 10.1051/e3sconf /202016406028

3. M. Aghajanpour, M.R. Nazer, Z. Obeidavi, M. Akbari, P. Ezati, N.M. Kor, American journal of cancer research 7(4) 740 (2017) PMID: 28469951

4. A. Lepeshkin, I. Shestopalova, L. Nadtochii, A. Chechetkina, V. Bespalov, D. Baranenko, V. Ilina, Agronomy Research 17(5), 1829-1838 (2019)

5. D.T. Silverman, C.A. Swanson, G. Gridley, S. Wacholder, L.M. Brown, R.B. Hayes, G.M. Swanson, Journal of the National Cancer Institute 90(22), 1710-1719 (1998) DOI: 10.1093/jnci/90.22.1710

6. P. Terry, A. Wolk, I. Persson, C. Magnusson, JAMA 285(23), 2975-2977 (2001) DOI: 10.1001/jama.285.23.2975

7. M. L. McCullough, A. S. Robertson, A. Chao, E. J. Jacobs, M. J. Stampfer, D.R. Jacobs, M. J. $\begin{array}{lllll}\text { Thun, Cancer Causes \& Control 14(10), 959-970 (2003) } & \end{array}$ DOI: 10.1023/B:CACO.0000007983.16045.a1

8. M.L. Neuhouser, R.E. Patterson, M.D. Thornquist, G.S. Omenn, I.B. King, G.E. Goodman, Cancer Epidemiology and Prevention Biomarkers 12(4), 350-358 (2003)

9. A. Steinbrecher, K. Nimptsch, A. Hüsing, S. Rohrmann, J. Linseisen, International journal of cancer 125(9), 2179-2186 (2009) DOI: 10.1002/ijc. 24555

10. S.M. Tortorella, S.G. Royce, P.V. Licciardi, T.C. Karagiannis, Antioxidants \& redox signaling 22(16), 1382-1424 (2015) DOI: 10.1089/ars.2014.6097

11. A.V. Snegovoy, A.I. Saltanov, L.V. Manzyuk, V.Y. Selchuk, Practical Oncology 10(1), 49-53 (2009)

12. D. Schrijvers, H. De Samblanx, F. Roila, ESMO Guidelines Working Group. Annals of oncology 21(5), v244-v247 (2010) DOI: 10.1093/annonc/mdq202

13. J.M. Facchini, E.P. Alves, C. Aguilera, R.M.M. Gern, M.L.L. Silveira, E. Wisbeck, S.A. Furlan, International journal of biological macromolecules 68, $72-77$ (2014) DOI: 10.1016/j.ijbiomac.2014.04.033

14. N.S. Osin, P.S. Popov, A.A. Borodulina, Patent no. 4039931/30-13 (1986)

15. I. Herr, M.W. Büchler, Cancer Treatment Rev 36(5), 377-383 (2010) DOI: 10.1016/j.ctrv.2010.01.002

16. X.Y. Cao, J.L. Liu, W. Yang, X. Hou, Q.J. Li, Molecular medicine reports 12(2), 23832389 (2015) DOI: 10.3892/mmr.2015.3648 